\title{
MIF's receptors revealed
}

\section{DOI:}

10.1038/nrd2341
The cytokine macrophage migration inhibitory factor (MIF) plays a crucial part in inflammatory diseases such as atherosclerosis but the receptors that underlie its involvement in such conditions remain unclear. Now, Bernhagen and colleagues, writing in Nature Medicine, have identified MIF as a ligand for the CXC-chemokine receptors CXCR2 and CXCR4, and suggest that targeting MIF could be used as an atherosclerotic therapy.

The authors first examined the in vitro effects of MIF on chemokine receptors. Surface-bound MIF induced arrest of primary human monocytes through CXCR2 and triggered the arrest of primary human effector T cells via CXCR4. Furthermore, CD74 (a MIF-binding protein) was implicated in CXCR2mediated MIF-induced arrest. To test whether MIF can directly elicit leukocyte chemotaxis through these receptors, the authors compared the promigratory effects of MIF on human blood mononuclear cell-derived monocytes expressing CXCR2 and $\mathrm{CD}^{+} \mathrm{T}$ lymphocytes expressing CXCR4. MIF induced chemotactic migration of both cell types, and, in monocytes, both CXCR2 and CD74 contributed to MIF-triggered chemotaxis. Biochemical assays revealed that MIF bound to CXCR2 and CXCR4 with nanamolar affinity, and elicited internalization of both receptors. Finally, it was shown that CXCR2 colocalizes with and physically interacts with CD74, demonstrating that a functional MIF receptor complex involves chemokine receptors and CD74.

Next, the in vivo actions of MIF were examined. The authors tested whether MIF acts via CXCR2 to induce monocyte recruitment in mice with early atherosclerotic endothelium. Monocyte arrest in carotid arteries of apolipoprotein E-deficient mice $\left(\right.$ Apoe $\left.^{-/-}\right)$fed a highfat diet was inhibited by antibodies to CXCR2, CD74 or MIF, indicating that MIF contributed to atherogenic recruitment via CXCR2 and CD74. A similar pattern of monocyte arrest was observed in arteries of wild-type mice treated with tumour-necrosis factor (TNF) to mimic acute vascular inflammation. The authors also measured accumulation of leukocytes in carotid arteries of $\mathrm{Mif}^{+/+}$and $\mathrm{Mif}^{/-}$ mice reconstituted with wild-type or $\mathrm{Il}_{\mathrm{r} \mathrm{rb}^{-/-} \text {bone marrow (Il8rb encodes }}$ CXCR2). After treatment with TNF, leukocyte accumulation was attenuated in mice lacking either or both Mif or Il8rb genes, providing further evidence that CXCR2 is required for MIF-mediated monocyte recruitment. In mice lacking the low-density lipoprotein receptor gene - a model of primary atherosclerosis - and the Mif gene, adhesion of monocytes to the luminal surface of aortic roots was reduced, and this was mirrored by a decrease in lesional macrophage content. Finally, antibody blockade

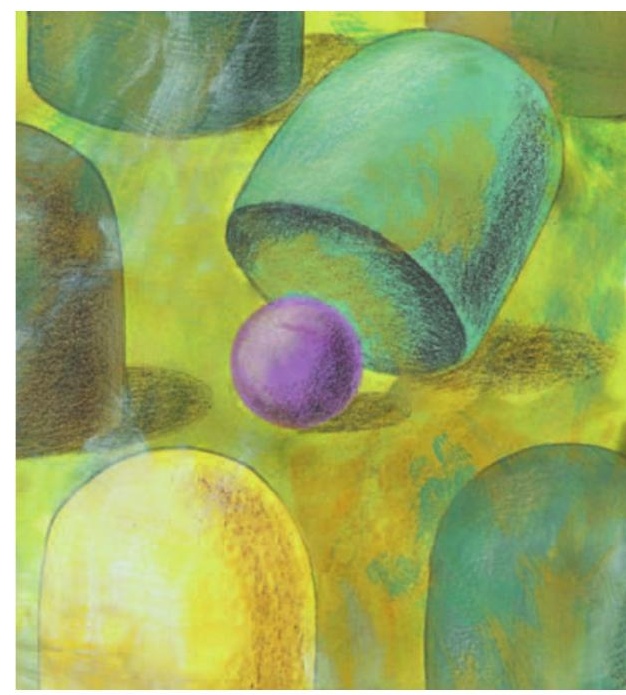

of MIF in Apoe $e^{-/-}$mice with severe lesions resulted in a reduced plaque area and plaque regression.

This study demonstrates that by activating both CXCR2 and CXCR4, MIF displays chemokine-like functions and acts as a regulator of inflammatory cell recruitment and atherogenesis. Therefore, targeting MIF could represent a new strategy to treat atherosclerosis.

Charlotte Harrison

ORIGINAL RESEARCH PAPER Bernhagen, J. et al. MIF is a noncognate ligand of CXC chemokine receptors in inflammatory and atherogenic cell recruitment. Nature Med. 13, 587-596 (2007)

FURTHER READING Morand, E. F, Leech, M. \& Bernhagen J. MIF: a new cytokine link between rheumatoid arthritis and atherosclerosis. Nature Rev. Drug Discov. 5, 399-411 (2006) 